\title{
Probing the partially localized supergravity background of the fundamental string ending on the Dp -brane
}

\author{
Donam Youm* \\ Theory Division, CERN, CH-1211, Geneva 23, Switzerland
}

(Received 1 July 1999; published 29 December 1999)

\begin{abstract}
We study the dynamics of the probe fundamental string in the field background of the partially localized supergravity solution for the fundamental string ending on the $\mathrm{D} p$-brane. We separately analyze the probe dynamics for its motion along the world volume direction and the transverse direction of the source $\mathrm{D} p$-brane. We compare the dynamics of the probe along the $\mathrm{D} p$-brane world volume direction to the bion dynamics.

PACS number(s): 11.15.Tk, 04.50.+h, 11.25.Sq, 11.27.+d
\end{abstract}

\section{INTRODUCTION}

The source and probe method has been useful for studying the bound states of branes. In this method, it is assumed that the source brane is much heavier than the probe brane. Thus, although the probe is under the influence of the fields produced by the source, the probe has no influence on the source field configuration. So, the source and probe system is described by the Dirac-Born-Infeld (DBI) or the NambuGoto (NB) action of the probe brane in the "static" field background of the source brane. Such a method has been successful in reproducing the brane intersection rules [1] and in studying some dynamics or statistical mechanics of branes, e.g., Refs. [2-6].

The source and probe method is useful especially when one wants to study intersecting brane configuration, since completely localized supergravity solutions for intersecting branes are not yet available. Note, for delocalized supergravity solutions, a constituent brane is not localized on the world volume of the other constituent. So, delocalized solutions are not useful for studying, for example, the dynamics of a brane constituent within the world volume of another brane constituent. For such a study, one lets one constituent be the probe and another constituent be the "static" background in which the probe moves. However, this method cannot be applied if one also wants to study the interaction among branes of the same type while these branes move in the background of brane of another type. It is the purpose of this paper to study such a case.

In Refs. [7-10], various types of (partially) localized supergravity solutions for intersecting branes in the core region of one constituent brane are constructed. For such solutions, one constituent is localized on the world volume of the other constituent (while the latter brane is delocalized on the former brane), provided that some of the overall transverse directions are delocalized in some cases. In this paper, we study the dynamics of the (localized) former type of brane (brane 1), which not only interacts with the (delocalized) other type of brane (brane 2) but also interacts with another brane 1. One can describe such dynamics with the DBI or the

\footnotetext{
*Email address: Donam.Youm@cern.ch
}

NG action of the brane 1 in the background field configuration of partially localized intersecting brane 1 and brane 2, where brane 1 is localized on brane 2 .

One can apply this method to study the dynamics for any type of intersecting brane configurations by using the partially localized intersecting brane solutions and applying the similar procedure as the work of this paper. But we will restrict our study to the case of the fundamental strings ending on the $\mathrm{D} p$-brane, because for this case the dynamics of the corresponding world volume solitons, i.e., the bions $[11,12]$, is relatively well understood, e.g., Refs. [13-15]. As we will see in the following section, we however find disagreement in dynamics of the probe fundamental string with the dynamics of bions. This seems to be due to the fact that the supergravity solutions for intersecting branes used in this paper are not fully localized ones. Namely, for the partially localized intersecting brane solutions used in this paper, the location of the $\mathrm{D} p$-brane along the longitudinal direction of the fundamental string is not specified, whereas the scalar field of the bion solution specifies such location. Also, it might be due to the difference in approximations used in the calculation of brane dynamics in the source-probe method and the world volume soliton method. However, the comparison of supergravity and world volume aspects of brane dynamics discussed in this paper may turn out to be useful in other relevant studies and when fully localized solutions are available.

The paper is organized as follows. In Sec. II, we summarize the partially localized supergravity solution for the fundamental string ending on the D p-brane. In Sec. III, we study the dynamics of the probe fundamental string in this supergravity background, closely following the previous works $[2,5]$ on the dynamics of the probe branes. We consider the cases where probe fundamental string moves along the longitudinal direction and the transverse direction of the source $\mathrm{D} p$-brane, separately.

\section{PARTIALLY LOCALIZED SUPERGRAVITY SOLUTION FOR THE FUNDAMENTAL STRING ENDING ON THE D $p$-BRANE}

In this section, we summarize the partially localized supergravity solution for the fundamental string ending on the 
$\mathrm{D} p$-brane ${ }^{1}$ constructed in Ref. [8]. The string-frame effective supergravity action for such configuration is given by

$$
\begin{aligned}
S= & \frac{1}{16 \pi G_{10}} \int d^{10} x \sqrt{-G}\left[e^{-2 \phi}\left(\mathcal{R}+4 \partial_{M} \phi \partial^{M} \phi-\frac{1}{12}\left|H_{3}\right|^{3}\right)\right. \\
& \left.-\frac{1}{2(p+2) !}\left|F_{p+2}\right|^{2}\right],
\end{aligned}
$$

where $G$ and $\mathcal{R}$ are respectively the determinant and the Ricci scalar of the spacetime metric $G_{M N}(M, N$ $=0,1, \ldots, 9)$ in the string frame, $\phi$ is the dilaton, $H_{3}$ is the field strength for the the 2-form potential $B_{M N}$ in the NeveuSchwarz-Neveu-Schwarz (NS-NS) sector, and $F_{p+2}$ is the field strength for the $(p+1)$-form potential $A_{M_{1} \cdots M_{p+1}}$ in the Ramond-Ramond (R-R) sector. For the $p=2$ case, there is an additional Chern-Simon term

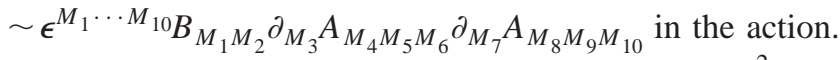
The supergravity solution has the following form: ${ }^{2}$

$$
\begin{gathered}
G_{M N} d x^{M} d x^{N}=-H_{F}^{-1} H_{p}^{-1 / 2} d t^{2}+H_{p}^{-1 / 2}\left(d x_{1}^{2}+\cdots+d x_{p}^{2}\right) \\
\times H_{F}^{-1} H_{p}^{1 / 2} d y^{2}+H_{p}^{1 / 2}\left(d z_{1}^{2}+\cdots+d z_{8-p}^{2}\right), \\
e^{\phi=} H_{F}^{-1 / 2} H_{p}^{(3-p) / 4}, \quad B_{t y}=-H_{F}^{-1} \\
A_{t x_{1} \cdots x_{p}}=-H_{p}^{-1}
\end{gathered}
$$

where the harmonic functions for the fundamental string and the D $p$-brane in the near horizon region $\left(\left|\vec{z}-\vec{z}_{0}\right| \approx 0\right)$ of the $\mathrm{D} p$-brane are respectively given by

$$
H_{F}=1+\sum_{i} \frac{Q_{i}}{\left[\left|\vec{x}-\vec{x}_{0 i}\right|^{2}+\frac{4 Q_{p}}{(p-4)^{2}}\left|\vec{z}-\vec{z}_{0}\right|^{p-4}\right]^{\left[(p-3)^{2}+3\right] / 2(p-4)}}, \quad H_{p}=\frac{Q_{p}}{\left|\vec{z}-\vec{z}_{0}\right|^{6-p}}
$$

Note, these harmonic functions describe the localized fundamental strings on the $\mathrm{D} p$-brane only for the $p=5$ case. (When $p=6$, the harmonic function $H_{p}$ is logarithmic and therefore this supergravity solution is not valid. For the $p$ $=7$ case, the spacetime is not asymptotically flat.) For the $p<5$ case, one has to delocalize $5-p$ of the overall transverse directions in order to localize the fundamental strings on the $\mathrm{D} p$-brane. Harmonic functions for this case are given by

$$
H_{F}=1+\sum_{i} \frac{Q_{i}}{\left[\left|\vec{x}-\vec{x}_{0 i}\right|^{2}+4 Q_{p}\left|\vec{z}-\vec{z}_{0}\right|\right]^{3}}, \quad H_{p}=\frac{Q_{p}}{\left|\vec{z}-\vec{z}_{0}\right|}
$$

Note, in the expressions for harmonic functions in Eq. (4), $\vec{z}$ is the part of overall transverse coordinates where the branes are localized. Namely, $\vec{z}$ in Eq. (4) is three-dimensional.

In this paper, we consider the case in which all the fundamental strings coincide at the origin of the $\mathrm{D} p$-brane world volume space (i.e., $\vec{x}_{0}=\overrightarrow{0}$, for all $i$ ) and the fundamental strings and the $\mathrm{D} p$-brane meet at the origin of the overall transverse space (i.e., $\vec{z}_{0}=\overrightarrow{0}$ ). The harmonic functions (3) in this case take the following forms:

\footnotetext{
${ }^{1}$ Of course, this supergravity solution does not strictly correspond to the fundamental string "ending", on $\mathrm{D} p$-brane, but rather corresponds to the fundamental string " piercing"' through $\mathrm{D} p$-brane. But at this moment, this supergravity solution is the closest that we have.
}

$$
H_{F}=\left\{\begin{array}{ll}
1+\frac{Q_{F}}{\left[x^{2}+4 Q_{p} z\right]^{3}}, & p<5, \\
1+\frac{Q_{F}}{\left[x^{2}+4 Q_{5} z\right]^{7 / 2}}, & p=5,
\end{array} \quad H_{p}=\frac{Q_{p}}{z},\right.
$$

where $x \equiv|\vec{x}|$ and $z \equiv|\vec{z}|$.

\section{DYNAMICS OF THE PROBE FUNDAMENTAL STRING}

In this section, we study the dynamics of the probe fundamental string that moves in the background of the source fundamental string ending on the source $\mathrm{D} p$-brane with the field configuration given by Eq. (2). We will assume that (i) the source brane is much heavier than the probe brane, i.e., there are large numbers of coinciding source fundamental strings and source $\mathrm{D} p$-branes, and (ii) the velocity of the probe fundamental string is very small and changes very slowly. Based on the first assumption, we neglect the back reaction on the source due to the moving probe. The second assumption implies that the radiation will be negligible, allowing quasistatic evolution of the system which is described by the geodesic motion in the moduli space.

The action for the probe fundamental string with the tension $T_{f}$ moving in the curved background is given by the following Nambu-Goto action:

\footnotetext{
${ }^{2}$ Similar class of solutions was first constructed in Ref. [7].
} 


$$
S=\int d \tau d \sigma L=-T_{f} \int d \tau d \sigma\left[\sqrt{-\operatorname{det} \hat{G}_{a b}}+\frac{1}{2 !} \epsilon^{a b} \hat{B}_{a b}\right],
$$

where $\hat{G}_{a b}$ and $\hat{B}_{a b}(a, b=\tau, \sigma)$ are respectively the pullbacks of the spacetime metric $G_{M N}$ and the NS-NS 2-form potential $B_{M N}$ to the world volume of the fundamental string: namely,

$$
\hat{G}_{a b} \equiv G_{M N} \partial_{a} X^{M} \partial_{b} X^{N}, \quad \hat{B}_{a b} \equiv B_{M N} \partial_{a} X^{M} \partial_{b} X^{N}
$$

In the static gauge $\left(X^{0}=\tau\right.$ and $X^{1}=\sigma$ with $X^{1}$ being the longitudinal coordinate of the fundamental string), the pullbacks take the following forms:

$$
\hat{G}_{a b}=G_{a b}+G_{i j} \partial_{a} X^{i} \partial_{b} X^{j}, \quad \hat{B}_{a b}=B_{a b}+B_{a j} \partial_{b} X^{j}+B_{i b} \partial_{a} X^{i}
$$

where scalars $X^{i}$ are the target space coordinates for the transverse space of the fundamental string.

Note, the above action (6) describes the probe fundamental string moving in the background of fields produced by the source brane configuration. Namely, $G_{M N}$ and $B_{M N}$ in Eqs. (6) and (7) are the fields in Eq. (2) produced by the source. Also, in the supergravity solution (2), the coordinates $\vec{x}, y$, and $\vec{z}$ correspond to the target space coordinates $\vec{X}$ of the probe fundamental string and are assumed to be functions of time $\tau=X^{0}$ only, i.e., $\vec{x}=\vec{x}(\tau)$. Namely, the probe fundamental string moves in the source background without oscillating. Therefore, the probe action (6) takes the following form:

$$
\begin{aligned}
S & =-T_{f} \int d \tau d \sigma\left[\sqrt{-\left(-H_{F}^{-1} H_{p}^{-1 / 2}+H_{p}^{-1 / 2} \mathrm{v}_{\|}^{2}+H_{p}^{1 / 2} \mathrm{v}_{\perp}^{2}\right) H_{F}^{-1} H_{p}^{1 / 2}}-H_{F}^{-1}\right] \\
& =-T_{f} \int d \tau d \sigma H_{F}^{-1}\left[\sqrt{1-H_{F} \mathrm{v}_{\|}^{2}-H_{p} H_{F} \mathrm{v}_{\perp}^{2}}-1\right] \\
& =-m_{f} \int d \tau H_{F}^{-1}\left[\sqrt{1-H_{F} \mathrm{v}_{\|}^{2}-H_{p} H_{F} \mathrm{v}_{\perp}^{2}}-1\right]
\end{aligned}
$$

where $\mathbf{V}_{\|}$and $\mathbf{V}_{\perp}$ are respectively the speeds of the probe fundamental string in the longitudinal direction $\vec{x}$ and the transverse direction $\vec{z}$ of the $\mathrm{D} p$-brane:

$$
\mathbf{V}_{\|} \equiv \sqrt{\sum_{i=1}^{p}\left(\frac{d x_{i}}{d \tau}\right)^{2}}, \quad \mathbf{v}_{\perp} \equiv \sqrt{\sum_{k=1}^{8-p}\left(\frac{d z_{k}}{d \tau}\right)^{2}}
$$

Since the configuration under consideration is assumed to be independent of the longitudinal coordinate $X^{1}=\sigma$ of the fundamental string, the integration (with the possible regularization) with respect to $\sigma$ in Eq. (9) just gives the volume factor, which combines with the tension $T_{f}$ of the probe fundamental string to give the mass $m_{f}$ of the probe fundamental string in the third line in Eq. (9). So, the above probe action $S$ effectively describes the dynamics of a test particle with mass $m_{f}$ moving in the background fields.

In the core region of the fundamental string and the D $p$-brane $(x \approx 0$ and $z \approx 0)$ or in the large source charge limit $\left(Q_{F} \gg 1\right.$ and $\left.Q_{p} \gg 1\right)$, the harmonic functions in the action (9) have the following forms:

$$
H_{F}=\left\{\begin{array}{ccc}
\frac{Q_{F}}{\left(x^{2}+4 Q_{p} z\right)^{3}}, & p<5, & \\
\frac{Q_{F}}{\left(x^{2}+4 Q_{5} z\right)^{7 / 2}}, & p=5, &
\end{array} H_{p}=\frac{Q_{p}}{z} .\right.
$$

In the case of the delocalized intersecting source configuration, the harmonic functions have the following forms:

$$
H_{F}=1+\frac{Q_{F}}{z^{6-p}}, \quad H_{p}=1+\frac{Q_{p}}{z^{6-p}},
$$

where the constant terms 1 in the harmonic functions are absent in the near horizon region $(z \approx 0)$. Note, in the partially localized case with the harmonic functions given by Eq. (11), unlike the delocalized case with the harmonic functions (12), the background geometry has the explicit dependence on the radial coordinate $x$ of the world volume space of the $\mathrm{D} p$-brane. This makes the study of nontrivial dynamics of the probe fundamental string in the relative transverse space possible.

So, explicitly in terms of the parameters of the source supergravity solution, the probe action (9) in the core region of the constituent source branes takes the following form:

$$
\begin{aligned}
S= & -m_{f} \int d \tau \frac{\left(x^{2}+4 Q_{p} z\right)^{n / 2}}{Q_{F}} \\
& \times\left[\sqrt{1-\frac{Q_{F} \mathbf{v}_{\|}^{2}}{\left(x^{2}+4 Q_{p} z\right)^{n / 2}}-\frac{Q_{p} Q_{F} \mathbf{v}_{\perp}^{2}}{z\left(x^{2}+4 Q_{p} z\right)^{n / 2}}-1}\right],
\end{aligned}
$$

where $n=6[n=7]$ for $p<5[p=5]$, and 


$$
S=-m_{f} \int d \tau \frac{z^{6-p}}{Q_{F}}\left[\sqrt{1-\frac{Q_{F} \mathrm{~V}_{\|}^{2}}{z^{6-p}}-\frac{Q_{p} Q_{F} \mathrm{v}_{\perp}^{2}}{z^{12-2 p}}}-1\right],
$$

for the delocalized case.

These actions for the probe fundamental string effectively describe the dynamics of a particle with mass $m_{f}$ moving in a velocity dependent potential. When the motion of the probe is restricted either to the relative transverse space (with the coordinates $\vec{x}$ ) or to the overall transverse space (with the coordinates $\vec{z}$ ) of the source brane configuration, the force on the particle becomes central, as well.

For the purpose of analyzing this motion, we set all the angular momenta of the probe except one in each space $\left(J_{\|}\right.$ $\neq 0$ in the relative transverse space and $J_{\perp} \neq 0$ in the overall transverse space) equal to zero. And one introduces the polar coordinates $\left(x, \theta_{\|}\right)$and $\left(z, \theta_{\perp}\right)$ in the rotation planes respectively associated with the angular momenta $J_{\|}$and $J_{\perp}$. Then, the velocities $\mathbf{V}_{\|}$and $\mathbf{v}_{\perp}$ in the relative transverse space and the overall transverse space, defined in Eq. (10), are decomposed as

$$
\mathrm{v}_{\|}^{2}=\dot{x}^{2}+x^{2} \dot{\theta}_{\|}^{2}, \quad \mathrm{v}_{\perp}^{2}=\dot{z}^{2}+z^{2} \dot{\theta}_{\perp}^{2}
$$

where the dot denotes the differentiation with respect to the time coordinate $\tau$.

Then, in general, the angular momenta $J_{\|}$and $J_{\perp}$ and the energy $E$ of the probe are given by

$$
\begin{gathered}
J_{\|}=p_{\theta_{\|}}=\frac{\partial L}{\partial \dot{\theta}_{\|}}=\frac{m_{f} x^{2} \dot{\theta}_{\|}}{\sqrt{1-H_{F} \mathrm{v}_{\|}^{2}-H_{p} H_{F} \mathrm{v}_{\perp}^{2}}}, \\
J_{\perp}=p_{\theta_{\perp}}=\frac{\partial L}{\partial \dot{\theta}_{\perp}}=\frac{m_{f} H_{p} z^{2} \dot{\theta}_{\perp}}{\sqrt{1-H_{F} \mathrm{v}_{\|}^{2}-H_{p} H_{F} \mathrm{v}_{\perp}^{2}}} \\
E=H=\frac{\partial L}{\partial \mathrm{v}_{\|}} \mathrm{v}_{\|}+\frac{\partial L}{\partial \mathrm{v}_{\perp}} \mathrm{v}_{\perp}-L \\
=\frac{m_{f}}{H_{F}}\left[\frac{1}{\sqrt{1-H_{F} \mathrm{v}_{\|}^{2}-H_{p} H_{F} \mathrm{v}_{\perp}^{2}}}\right]
\end{gathered}
$$

So, explicitly the expressions for the angular momenta and the energy of the probe fundamental string in each case are as follows:

$$
\begin{gathered}
J_{\|}=\frac{m_{f} x^{2} \dot{\theta}_{\|}}{\sqrt{1-\frac{Q_{F} \mathrm{v}_{\|}^{2}}{\left(x^{2}+4 Q_{p} z\right)^{n / 2}}-\frac{Q_{p} Q_{F} \mathrm{v}_{\perp}^{2}}{z\left(x^{2}+4 Q_{p} z\right)^{n / 2}}}}, \\
J_{\perp}=\frac{m_{f} Q_{p} z \dot{\theta}_{\perp}}{\sqrt{1-\frac{Q_{F} \mathrm{v}_{\|}^{2}}{\left(x^{2}+4 Q_{p} z\right)^{n / 2}}-\frac{Q_{p} Q_{F} \mathrm{v}_{\perp}^{2}}{z\left(x^{2}+4 Q_{p} z\right)^{n / 2}}}},
\end{gathered}
$$

$$
\begin{aligned}
E= & \frac{m_{f}\left(x^{2}+4 Q_{p} z\right)^{n / 2}}{Q_{F}} \\
& \times\left[\frac{1}{\sqrt{1-\frac{Q_{F} \mathbf{v}_{\|}^{2}}{\left(x^{2}+4 Q_{p} z\right)^{n / 2}}-\frac{Q_{p} Q_{F} \mathbf{v}_{\perp}^{2}}{z\left(x^{2}+4 Q_{p} z\right)^{n / 2}}}}-1\right],
\end{aligned}
$$

where $n=6[n=7]$ for $p<5 \quad[p=5]$, and

$$
\begin{aligned}
J_{\|}= & \frac{m_{f} x^{2} \dot{\theta}_{\|}}{\sqrt{1-\frac{Q_{F} \mathbf{v}_{\|}^{2}}{z^{6-p}}-\frac{Q_{p} Q_{F} \mathrm{v}_{\perp}^{2}}{z^{12-2 p}}}}, \\
J_{\perp}= & \frac{m_{f} Q_{p} z^{p-4} \dot{\theta}_{\perp}}{\sqrt{1-\frac{Q_{F} \mathbf{v}_{\|}^{2}}{z^{6-p}}-\frac{Q_{p} Q_{F} \mathbf{v}_{\perp}^{2}}{z^{12-2 p}}}} \\
E & \left.=\frac{m_{f} z^{6-p}\left[\frac { 1 } { Q _ { F } } \left[\sqrt{1-\frac{Q_{F} \mathbf{v}_{\|}^{2}}{z^{6-p}}-\frac{Q_{p} Q_{F} \mathbf{v}_{\perp}^{2}}{z^{12-2 p}}}\right.\right.}{\sqrt{1}}\right],
\end{aligned}
$$

for the delocalized case.

\section{A. The motion of the probe fundamental string in the relative transverse space}

In this subsection, we study the dynamics of the probe fundamental string whose motion is restricted to the relative transverse space of the source brane configuration. In this case, $v_{\perp}=0$ and the coordinate $z$ is constant in time. Generally, the angular momentum $J_{\|}$in the relative transverse space and the energy $E$ of the probe fundamental string have the following forms:

$$
J_{\|}=\frac{m_{f} x^{2} \dot{\theta}_{\|}}{\sqrt{1-H_{F} \mathbf{v}_{\|}^{2}}}, \quad E=\frac{m_{f}}{H_{F}}\left[\frac{1}{\sqrt{1-H_{F} \mathbf{v}_{\|}^{2}}}-1\right] .
$$

So, explicitly the angular momentum and the energy for each case are as follows:

$$
\begin{aligned}
& J_{\|}=\frac{m_{f} x^{2} \dot{\theta}_{\|}}{\sqrt{1-\frac{Q_{F} \mathrm{v}_{\|}^{2}}{\left(x^{2}+4 Q_{p} z\right)^{n / 2}}},} \\
& E=\frac{m_{f}\left(x^{2}+4 Q_{p} z\right)^{n / 2}}{Q_{F}}\left[\frac{1}{\sqrt{1-\frac{Q_{F} \mathrm{v}_{\|}^{2}}{\left(x^{2}+4 Q_{p} z\right)^{n / 2}}}}-1\right],
\end{aligned}
$$


where $n=6[n=7]$ for $p<5 \quad[p=5]$, and

$$
\begin{aligned}
& J_{\|}=\frac{m_{f} x^{2} \dot{\theta}_{\|}}{\sqrt{1-\frac{Q_{F} \mathbf{v}_{\|}^{2}}{z^{6-p}}}}, \\
& E=\frac{m_{f} z^{6-p}}{Q_{F}}\left[\frac{1}{\sqrt{1-\frac{Q_{F} \mathbf{v}_{\|}^{2}}{z^{6-p}}}}-1\right],
\end{aligned}
$$

for the delocalized case.

From the above expression for the energy $E$ of the probe fundamental string, one obtains the following kinetic relation:

$$
E=\frac{1}{2} m_{f} \mathbf{v}_{\|}^{2}+W(x), \quad W(x)=E\left[1-\frac{1+\frac{E}{2 m_{f}} H_{F}}{\left(1+\frac{E}{m_{f}} H_{F}\right)^{2}}\right],
$$

where the harmonic function $H_{F}$ is given by Eq. (11) or Eq. (12). By further using the expression for the angular momentum $J_{\|}$in Eq. (19), one obtains the following kinetic relation for the radial motion of the probe:

$$
\begin{aligned}
E & =\frac{1}{2} m_{f} \dot{x}^{2}+V(x) \\
V(x) & =E\left[1-\frac{1+\frac{E}{2 m_{f}} H_{F}}{\left(1+\frac{E}{m_{f}} H_{F}\right)^{2}}\right]+\frac{J_{\|}^{2}}{2 m_{f} x^{2}} \frac{1}{\left(1+\frac{E}{m_{f}} H_{F}\right)^{2}} .
\end{aligned}
$$

So, the radial motion (along the $x$ direction) of the probe is that of the test particle with mass $m_{f}$ moving in a velocityindependent central force potential $V(x)$.

In the partially localized case, the effective potential $V(x)$ is explicitly given by

$$
\begin{aligned}
V(x)=E\left[1-\frac{1+\frac{b^{n}}{2\left(x^{2}+4 Q_{p} z\right)^{n / 2}}}{\left(1+\frac{b^{n}}{\left(x^{2}+4 Q_{p} z\right)^{n / 2}}\right)^{2}}\right] \\
+\frac{J_{\|}^{2}}{2 m_{f} x^{2}} \frac{1}{\left(1+\frac{b^{n}}{\left(x^{2}+4 Q_{p} z\right)^{n / 2}}\right)^{2}},
\end{aligned}
$$

where $b$ is the characteristic scale given by

$$
b=\left(\frac{E Q_{F}}{m_{f}}\right)^{1 / n}
$$

where $n=6[n=7]$ for $p<5[p=5]$.

The dynamics of the probe fundamental string along the radial direction $x$ can be studied by analyzing an effective velocity-dependent central force potential $V(x)$ in Eq. (23). In the "delocalized"' source background with the harmonic function $H_{F}$ in Eq. (12) being independent of the radial coordinate $x$, the dynamics of the probe fundamental string in the world volume space of the $\mathrm{D} p$-brane is trivial: the only force on the probe is the repulsive centrifugal force due to nonzero angular momentum $J_{\|}$of the probe. However, with the partially localized source background, one can study nontrivial dynamics of the probe fundamental string since the source fundamental string is now localized on the source $\mathrm{D} p$-brane and therefore the effective potential $V(x)$ in Eq. (24) has explicit dependence on $x$.

At large distance $x \gg b$ from the source fundamental string, the effective potential in Eq. (24) takes the following form:

$$
V_{x \gg b} \rightarrow \frac{3 E^{2} Q_{F}}{2 m_{f}\left(x^{2}+4 Q_{p} z\right)^{n / 2}}+\frac{J_{\|}^{2}}{2 m_{f} x^{2}} .
$$

So, the motion of the probe fundamental string is qualitatively similar to the motion in the background of the source fundamental string only, except that the strength of the repulsive potential [the first term in Eq. (26)] is decreased due to the presence of the source $\mathrm{D} p$-brane. This contribution from the source $\mathrm{D} p$-brane gets enhanced for the larger D $p$-brane charge $Q_{p}$ and at larger distance $z$ from the source $\mathrm{D} p$-brane. However, the centrifugal potential (the second term) remains the same regardless of the presence of the source D $p$-brane.

At short distance $x \ll b$ from the source fundamental string and very close to the source $\mathrm{D} p$-brane $\left(z \ll b^{2} / Q_{p}\right)$, the effective potential is approximated to

$$
\begin{aligned}
V_{x \ll b, z \ll b^{2} / Q_{p}} \rightarrow & E-\frac{m_{f}}{2 Q_{F}}\left(x^{2}+4 Q_{p} z\right)^{n / 2} \\
& +\frac{m_{f} J_{\|}^{2}}{2 E^{2} Q_{F}^{2}} \frac{\left(x^{2}+4 Q_{p} z\right)^{n}}{x^{2}} .
\end{aligned}
$$

The (energy $E$ independent) repulsive potential term is enhanced again due to the presence of the source $\mathrm{D} p$-brane: for larger $Q_{p}$ and $z$, the repulsive force becomes stronger. Unlike the case of the long distance region, the probe fundamental string now feels the effect of the source $\mathrm{D} p$-brane on the centrifugal potential when the probe fundamental string gets very close to the source.

We now discuss the motion of the probe fundamental string in the source background. The turning points, where the radial velocity $\dot{x}$ of the probe becomes zero, are located at the values of $x$ where $E=V(x)[\mathrm{cf}$. Eq. (23)] and therefore are the roots of the following equation:

$$
1+\frac{b^{n}}{2\left(x^{2}+4 Q_{p} z\right)^{n / 2}}=\frac{b_{*}^{2}}{x^{2}},
$$


where $b$ is given in Eq. (25), $b_{*} \equiv J_{\|} / \sqrt{2 m_{f} E}$ and again $n$ $=6[n=7]$ for $p<5 \quad[p=5]$. This equation always has one positive root $x$ for nonzero angular momentum $J_{\|}$of the probe. And when $J_{\|}=0$, there is no root $x$ for this equation. Furthermore, the radial force $F(x)=-d V(x) / d x$ on the probe diverges as $x \rightarrow 0$, when $J_{\|} \neq 0$. On the other hand, when the angular momentum $J_{\|}$is zero, the force vanishes at $x=0$. So, the motion of the probe fundamental string can be summarized as follows. When the probe has nonzero angular momentum, the probe will always be scattered away when it reaches the source fundamental string. When the probe has no angular momentum, it will eventually be absorbed by the source.

In the following, we compare the dynamics of the bions, which was previously studied in Refs. [13-15], to the dynamics of the probe fundamental string along the world volume direction of the D-brane studied in the above paragraphs. It is natural to expect that these two systems have the same dynamics, since the bions in the $(p+1)$-dimensional DBI theory are interpreted as the ends of fundamental strings on the $\mathrm{D} p$-brane in the very weak string coupling limit $\left(g_{s}\right.$ $\rightarrow 0$ ). Namely, the motion of the ends of the fundamental strings on the D-brane along the D-brane world volume direction is essentially the motion of the bions. However, as we shall see in the following, our description of dynamics of the probe fundamental string studied in the above is too simplified to reproduce the dynamics of the world volume solitons, i.e., bions.

In the following, we summarize the dynamics of the bions, studied in Refs. [13-15], for the purpose of comparing the dynamics of bions to the dynamics of the probe and for the purpose of fixing the notations. The $(p+1)$-dimensional DBI action has the following form:

$$
S_{\mathrm{DBI}}=-\int d^{p+1} \sigma \sqrt{-\operatorname{det}\left(\eta_{M N} \partial_{\mu} X^{M} \partial_{\nu} X^{N}+F_{\mu \nu}\right)},
$$

where $\eta_{M N}(M, N=0,1, \ldots, 9)$ is the metric for the Minkowskian target space and $F_{\mu \nu}=\partial_{\mu} A_{\nu}-\partial_{\nu} A_{\mu}(\mu, \nu$ $=0,1, \ldots, p)$ is the field strength of the world volume $U(1)$ gauge field $A_{\mu}$. In the "static" gauge, in which the world volume coordinates $\sigma^{\mu}$ are identified with the target space coordinates as $\sigma^{\mu}=X^{\mu}\left[\left(X^{M}\right)=\left(X^{\mu}, X^{m}\right)\right.$ with $m=p$ $+1, \ldots, 9]$, the DBI action (29) takes the following form:

$$
S_{\mathrm{DBI}}=-\int d^{p+1} \sigma \sqrt{-\operatorname{det}\left(\eta_{\mu \nu}+\partial_{\mu} X^{m} \partial_{\nu} X^{m}+F_{\mu \nu}\right)} .
$$

The ends of the fundamental strings on the $\mathrm{D} p$-brane world volume correspond to the bions which carry the electric charge of the world volume $U(1)$ gauge field $A_{\mu}$. One scalar, say $X:=X^{p+1}$, associated with the longitudinal direction of the fundamental string in the target space is nontrivial. In general, the bion solution has the following form $[11,12]$ :

$$
A_{0}=-H, \quad X=H ; \quad H=\sum_{k} \frac{q_{k}}{\left|\vec{\sigma}-\vec{x}_{k}\right|^{p-2}},
$$

where $\vec{\sigma}=\left(\sigma^{1}, \ldots, \sigma^{p}\right)$ is the spatial components of the world volume coordinates $\left(\sigma^{\mu}\right)=(\tau, \vec{\sigma})$ and $\vec{x}_{k}$ is the location of a bion with the electric charge $q_{k}$ in the world volume space.

Since we are interested in the low velocity dynamics of bions, it is sufficient to consider the following linearized approximation to the DBI action (30):

$$
\begin{aligned}
S_{\mathrm{DBI}} & \approx \frac{1}{2} \int d^{p+1} \sigma\left[\delta_{m n} \eta^{\mu \nu} \partial_{\mu} X^{m} \partial_{\nu} X^{n}+\frac{1}{2} F_{\mu \nu} F^{\mu \nu}\right] \\
& =\frac{1}{2} \int d^{p+1} \sigma\left[\eta^{\mu \nu} \partial_{\mu} X \partial_{\nu} X+\frac{1}{2} F_{\mu \nu} F^{\mu \nu}\right],
\end{aligned}
$$

where in the second line only one scalar $X$ associated with the longitudinal direction of the attached fundamental string is kept.

To study the dynamics of the bions, we allow the locations of the bions to change with time, i.e., $\vec{x}_{k}=\vec{x}_{k}(\tau)$. So, $\overrightarrow{\mathrm{v}}_{k}=d \vec{x}_{k}(\tau) / d \tau$ is the velocity of the $k$ th bion with the electric charge $q_{k}$. One has to also add the following source term $S_{\text {source }}$ for the BI $U(1)$ field $A_{\mu}$ and scalar field $X$, and the free term $S_{\text {free }}$ for the bions:

$$
\begin{aligned}
S_{\text {source }} & =(2-p) \Omega_{p-1} \sum_{k} \int d \tau\left[q_{k} X \sqrt{1-\mathrm{v}_{k}^{2}}+q_{k} A_{\mu} \frac{\partial x_{k}^{\mu}}{\partial \tau}\right], \\
S_{\text {free }} & =-\sum_{k} \int d \tau m_{k}(\epsilon) \sqrt{1-\mathrm{v}_{k}^{2}},
\end{aligned}
$$

where $\Omega_{p-1}=2 \pi^{p / 2} / \Gamma(p / 2)$ is the volume of the unit ( $p$ $-1)$-sphere $S^{p-1}$ and $m_{k}(\epsilon)$ is the "regularized" mass of the $k$ th bion. Here, $\epsilon$ is the cutoff for the radial distance from the bions, i.e., we restrict ourselves to the region $\left|\vec{\sigma}-\vec{x}_{k}\right|$ $\geqslant \epsilon$. Then, $m_{k}(\epsilon)$ corresponds to the mass of the fundamental string whose length is truncated due to the regularization [11]. The source term can be interpreted as being related to the bulk supergravity configuration. Namely, the D $p$-brane, whose shape in the $(x, X)$ plane is given by $X(x)$, is the source of the first term in $S_{\text {source }}$, and the end of the fundamental string on the Dp-brane is the source of the world volume $U(1)$ gauge field $A_{\mu}$ (the second term in $S_{\text {source }}$ describes such coupling). $S_{\text {free }}$ is the action for the bions with masses $m_{k}(\boldsymbol{\epsilon})$.

Note, since the bions, which carry electric charges, have nonzero velocities, the magnetic field is induced and the velocity dependent force will also be induced. To study such and other effects on the bion dynamics due to nonzero velocities, one perturbs the fields around the static configuration (31). Then, one substitutes the perturbed fields, which satisfy the equations of motion to the order $\mathcal{O}\left(\mathrm{v}^{2}\right)$ in the velocity $\vee$ of the bions, into the action $S=S_{\mathrm{DBI}}+S_{\text {source }}$ $+S_{\text {free }}$ in order to obtain the following on-shell effective action for the bions to the order $\mathcal{O}\left(\mathrm{v}^{2}\right)$ [13-15]: 


$$
\begin{aligned}
S \approx & \frac{p-2}{2} \Omega_{p-1} \int d \tau\left[\sum_{k} m_{k}(\boldsymbol{\epsilon}) \mathrm{v}_{k}^{2}+(p-2) \Omega_{p-1}\right. \\
& \left.\times \sum_{k<l} q_{k} q_{l} \frac{\left|\overrightarrow{\mathbf{v}}_{k}-\overrightarrow{\mathbf{v}}_{l}\right|^{2}}{\left|\vec{x}_{k}-\vec{x}_{l}\right|^{p-2}}\right],
\end{aligned}
$$

for large separations $\left|\vec{x}_{k}-\vec{x}_{l}\right| \gg 0$ for bions.

The system of the probe fundamental string [with the action (6)] moving in the source background, given by Eq. (2) with Eq. (5), is the bulk counterpart to the dynamic system of two bions in the $(p+1)$-dimensional DBI theory. In this case, the indices $k$ and $l$ in Eqs. (31) and (34) run from 1 to 2. We let the first [second] bion correspond to the probe [the source] fundamental string. This is a two-body system under a central force. Such system can be reduced to an equivalent one-body system by replacing the positions $\vec{x}_{1}$ and $\vec{x}_{2}$ of the bions by their center-of-mass position $\vec{R}=\left(m_{1} \vec{x}_{1}\right.$ $\left.+m_{2} \vec{x}_{2}\right) /\left(m_{1}+m_{2}\right)$ and relative position $\vec{r}=\vec{x}_{1}-\vec{x}_{2}$. The action (34) then transforms to the following form:

$$
S \approx \frac{p-2}{2} \Omega_{p-1} \int d \tau\left[M V^{2}+\mu \mathrm{V}^{2}+(p-2) \Omega_{p-1} \frac{q_{1} q_{2} \mathrm{v}^{2}}{r^{p-2}}\right],
$$

where $M=m_{1}+m_{2}$ is the total mass, $\mu=m_{1} m_{2} / M$ is the reduced mass, $V=|d \vec{R} / d \tau|$ is the center-of-mass velocity, and $\mathrm{v}=|d \vec{r} / d \tau|$ is the relative velocity. Thus the motion of bions is described by the geodesic motion in the moduli space with the following metric:

$$
d s_{M S}^{2}=M d \vec{R}^{2}+\left[\mu+(p-2) \Omega_{p-1} \frac{q_{1} q_{2}}{r^{p-2}}\right] d \vec{r}^{2}
$$

As expected, the center-of-mass moves freely but the relative motion of the bions is under the influence of a velocity dependent central potential. Since the source fundamental string is assumed to be much heavier than the probe fundamental string, the second bion is much heavier than the first bion, i.e., $m_{1} \ll m_{2}$. Then, the action (35) in the center-ofmass coordinate system $(\vec{V}=\overrightarrow{0})$ is approximated to ${ }^{3}$

$$
S \approx \frac{p-2}{2} \Omega_{p-1} \int d \tau\left[m_{1} \mathrm{v}_{1}^{2}+(p-2) \Omega_{p-1} \frac{q_{1} q_{2} \mathrm{v}_{1}^{2}}{r^{p-2}}\right],
$$

which describes dynamics of the first bion in the background of the second bion, which is fixed in space.

In order to compare the above result for the bion dynamics to the bulk theory result, one has to obtain the velocity dependent potential from the energy $E$ of the probe fundamental string in Eq. (20). In the limit of very small probe

\footnotetext{
${ }^{3}$ When $m_{1} \ll m_{2}$, the quantities in the center-of-mass frame are approximated as $\vec{x}_{1}=\left[m_{2} /\left(m_{1}+m_{2}\right)\right] \vec{r} \approx \vec{r}$ and $\mu=m_{1} m_{2} /\left(m_{1}\right.$ $\left.+m_{2}\right) \approx m_{1}$.
}

velocity $\left(\mathrm{V}_{\|} \approx 0\right)$, the energy $E$ of the probe fundamental string in Eq. (20) is expanded in powers of the velocity as

$$
E=\frac{1}{2} m_{f} \mathbf{v}_{\|}^{2}+\frac{3 m_{f} Q_{F} \mathbf{v}_{\|}^{4}}{8\left(x^{2}+4 Q_{p} z\right)^{n / 2}}+\frac{5 m_{f} Q_{F}^{2} \mathbf{v}_{\|}^{6}}{16\left(x^{2}+4 Q_{p} z\right)^{n}}+\cdots,
$$

and, therefore, to the leading order in $\mathbf{v}_{\|}$, the velocity dependent potential on the probe is

$$
V_{\mathrm{eff}} \approx \frac{3 m_{f} Q_{F} \mathbf{v}_{\|}^{4}}{8\left(x^{2}+4 Q_{p} z\right)^{n / 2}} .
$$

This expression for the effective potential on the probe fundamental string has different dependence on the velocity $\mathbf{V}_{\|}$and the radial coordinate $x$ from the effective potential on the light bion given in Eq. (37). In addition, one also finds disagreement of the probe moduli metric, which describes the geodesic motion of the probe fundamental string, with the moduli metric (36) of bions, as we see in the following. In the limit of very small probe velocity $\left(\mathrm{V}_{\|} \approx 0\right.$ and $\mathrm{V}_{\perp}$ $\approx 0$ ), the on-shell action $(9)$ is approximated to

$$
S \approx \frac{1}{2} m_{f} \int d \tau\left(\mathrm{v}_{\|}^{2}+H_{p} \mathrm{v}_{\perp}^{2}\right)
$$

to the lowest order in the velocities. The vanishing of the static potential in this on-shell action is in accordance with the fact that we are considering a BPS configuration. From the definitions of the probe fundamental string velocities (10), one can see that the moduli metric of the probe fundamental string is given by

$$
d s_{F}^{2}=d x_{i} d x_{i}+H_{p} d z_{k} d z_{k}
$$

This moduli metric implies that the probe moves freely along the world volume directions of the $\mathrm{D} p$-brane, whereas the moduli metric (36) for the bions describes the motion under the influence of the velocity dependent central potential.

This disagreement may be traced from the following factors. First, in calculating the effective action for the probe fundamental string, we assumed that the field configurations are static, uninfluenced by the moving probe fundamental string. As was done originally in Refs. [16-20], when one studies the motion of collection of interacting solitons (in the low-velocity limit), which is described by the geodesic motion in the moduli space, one usually takes into account the perturbation (in a slow-motion expansion) of the original static fields due to nonzero velocities of the solitons. This is properly done in the case of the interaction of bions in the above, but not in the case of the probe fundamental string moving in the source background. Namely, in the sourceprobe method, one assumes that the source is much heavier than the probe and therefore the source is uninfluenced by the probe. On the other hand, in the case of bion dynamics, first we assumed that all the bions have comparable mass (therefore, the field produced by one bion is influenced by those of other bions) and at the end we let one of the bions be much heavier than the others. Second, the supergravity 
background (2) in which the probe fundamental string moves corresponds to the configuration where the source $\mathrm{D} p$-brane is delocalized along the longitudinal direction (the $y$ direction) of the source fundamental string. On the other hand, as for the bion solution in Eq. (31), the location of the $\mathrm{D} p$-brane along the longitudinal direction of the fundamental string is specified by the scalar $X$. So, although the partially localized supergravity solution (2) has all the parameters of the bions, i.e., the charges and the locations of the bions, it still lacks one special feature of the bion solution that a scalar $X$ of the bion solution specifies the location or the shape of the $\mathrm{D} p$-brane along the longitudinal direction of the fundamental string. In the fully localized supergravity solution, one would expect to see the shape of the D $p$-brane pulled by the fundamental string, just like the case of the bion solution. In the case of the configurations describing one type of brane within the world volume of another type of brane, we will not encounter this problem, since there is no relative transverse direction that is delocalized. If one properly takes into account the above observations, it might be possible to reproduce the moduli metric for the bion interaction by studying probe fundamental string moving in the source background of the fundamental strings ending on the $\mathrm{D} p$-brane.

\section{B. The motion of the probe fundamental string in the overall transverse space}

In this subsection, we study the dynamics of the probe fundamental string whose motion is restricted to the overall transverse space. In this case, $\mathbf{v}_{\|}=0$ and the coordinate $x$ is constant in time. Generally, the angular momentum $J_{\perp}$ in the overall transverse space and the energy $E$ of the probe fundamental string have the following forms:

$$
J_{\perp}=\frac{m_{f} H_{p} z^{2} \dot{\theta}_{\perp}}{\sqrt{1-H_{p} H_{F} \mathrm{v}_{\perp}^{2}}}, \quad E=\frac{m_{f}}{H_{F}}\left[\frac{1}{\sqrt{1-H_{p} H_{F} \mathrm{v}_{\perp}^{2}}}-1\right] .
$$

So, the explicit expressions for the angular momentum and the energy are

$$
\begin{aligned}
J_{\perp} & =\frac{m_{f} Q_{p} z \dot{\theta}_{\perp}}{\sqrt{1-\frac{Q_{p} Q_{F} \mathrm{v}_{\perp}^{2}}{z\left(x^{2}+4 Q_{p} z\right)^{n / 2}}}}, \\
E & =\frac{m_{f}\left(x^{2}+4 Q_{p} z\right)^{n / 2}}{Q_{F}}\left[\frac{1}{\sqrt{1-\frac{Q_{p} Q_{F} \mathrm{v}_{\perp}^{2}}{z\left(x^{2}+4 Q_{p} z\right)^{n / 2}}}}-1\right],
\end{aligned}
$$

where $n=6[n=7]$ for $p<5[p=5]$, and

$$
\begin{aligned}
J_{\perp} & =\frac{m_{f} Q_{p} z^{p-4} \dot{\theta}_{\perp}}{\sqrt{1-\frac{Q_{p} Q_{F} \mathrm{v}_{\perp}^{2}}{z^{12-2 p}}}} \\
E & =\frac{m_{f} z^{6-p}}{Q_{F}}\left[\frac{1}{\sqrt{1-\frac{Q_{p} Q_{F} \mathrm{v}_{\perp}^{2}}{z^{12-2 p}}}}-1\right],
\end{aligned}
$$

for the delocalized case.

From the above expression for the energy $E$ of the probe fundamental string, one obtains the following kinetic relation:

$$
E=\frac{1}{2} m_{f} v_{\perp}^{2}+W(z), \quad W(z)=E\left[1-\frac{1}{H_{p}} \frac{1+\frac{E}{2 m_{f}} H_{F}}{\left(1+\frac{E}{m_{f}} H_{F}\right)^{2}}\right],
$$

where the harmonic functions $H_{F}$ and $H_{p}$ are given in Eqs. (11) and (12). Further using the expression for the angular momentum $J_{\perp}$ in Eq. (42), one obtains the following kinetic relation for the radial motion of the probe:

$$
\begin{aligned}
& E= \frac{1}{2} m_{f} \dot{z}^{2}+V(z), \\
& V(z)=E\left[1-\frac{1}{H_{p}} \frac{1+\frac{E}{2 m_{f}} H_{F}}{\left(1+\frac{E}{m_{f}} H_{F}\right)^{2}}\right] \\
&+\frac{J_{\perp}^{2}}{2 m_{f} H_{p}^{2} z^{2}} \frac{1}{\left(1+\frac{E}{m_{f}} H_{F}\right)^{2}} .
\end{aligned}
$$

So, again the radial motion (along the $z$ direction) of the probe fundamental string is that of a test particle with mass $m_{f}$ moving in an effective velocity-independent central force potential $V(z)$.

We notice the qualitative difference in the effective potential (therefore, the qualitative difference in the dynamics of the probe) between this case and the case of the dynamics in the relative transverse space of the source brane configuration with the effective potential given in Eq. (23). Namely, whereas the effective potential for the dynamics in the world volume direction of the source $\mathrm{D} p$-brane is affected by the source $\mathrm{D} p$-brane only through the $\mathrm{D} p$-brane charge $Q_{p}$ in the harmonic function $H_{F}$ for the source fundamental string, in the case of the dynamics in the directions transverse to the $\mathrm{D} p$-brane the effective potential explicitly depends on the harmonic function $H_{p}$ of the $\mathrm{D} p$-brane. This is expected 
from the fact that our supergravity field background (2) for the source is delocalized along the longitudinal direction of the fundamental string: the probe fundamental string will feel the uniform force field of the same strength (produced by the source $\mathrm{D} p$-brane) as it moves along the $\mathrm{D} p$-brane world volume direction. However, the source $\mathrm{D} p$-brane is in fact pulled by the source fundamental string and therefore the probe fundamental string will feel the varying force field of the source $\mathrm{D} p$-brane and ultimately hit the $\mathrm{D} p$-brane as it moves towards the source fundamental string along the $\mathrm{D} p$-brane world volume direction. This force on the probe fundamental string due to the source $\mathrm{D} p$-brane is the bulk counterpart to the force on the bion due to the scalar charge of $X$ in Eq. (31). This is one of the reasons for the mismatch of the probe dynamics in the $\mathrm{D} p$-brane world volume direction and the bion dynamics, as pointed out in the previous section.

For the partially localized case, the effective potential in the core region is explicitly given by

$$
\begin{aligned}
V(z)= & {\left[1-\frac{z}{Q_{p}} \frac{1+\frac{\left(Q_{p} b\right)^{n / 2}}{2\left(x^{2}+4 Q_{p} z\right)^{n / 2}}}{\left(1+\frac{\left(Q_{p} b\right)^{n / 2}}{\left(x^{2}+4 Q_{p} z\right)^{n / 2}}\right)^{2}}\right] } \\
& +\frac{J_{\perp}^{2}}{2 m_{f} Q_{p}^{2}} \frac{1}{\left(1+\frac{\left(Q_{p} b\right)^{n / 2}}{\left(x^{2}+4 Q_{p} z\right)^{n / 2}}\right)^{2}},
\end{aligned}
$$

where the characteristic scale $b$ has the following form:

$$
b=\left(\frac{E Q_{F}}{m_{f} Q_{p}^{n / 2}}\right)^{2 / n},
$$

and for the delocalized case,

$$
\begin{aligned}
V(z)= & E\left[1-\frac{z^{6-p}}{Q_{p}} \frac{1+\frac{b^{6-p}}{2 z^{6-p}}}{\left(1+\frac{b^{6-p}}{z^{6-p}}\right)^{2}}\right] \\
& +\frac{J_{\perp}^{2} z^{10-2 p}}{2 m_{f} Q_{p}^{2}} \frac{1}{\left(1+\frac{b^{6-p}}{z^{6-p}}\right)^{2}},
\end{aligned}
$$

where the characteristic scale $b$ is given by

$$
b=\left(\frac{E Q_{F}}{m_{f}}\right)^{1 /(6-p)} .
$$

We now analyze the dynamics of the probe fundamental string moving along the radial direction $z$ of the overall transverse space. The dynamics is nontrivial for both delocalized and partially localized cases. We study both of these cases and compare the differences.

At large distance $z \gg b$ from the source, the effective potential $V(z)$ in Eq. (46) takes the following form:

$$
\begin{gathered}
V_{z \gg b} \rightarrow E\left[1-\frac{z}{Q_{p}}\left(1-\frac{3 E Q_{F}}{2 m_{f}\left(x^{2}+4 Q_{p} z\right)^{n / 2}}\right)\right] \\
+\frac{J_{\perp}^{2}}{2 m_{f} Q_{p}^{2}}\left[1-\frac{2 E Q_{F}}{m_{f}\left(x^{2}+4 Q_{p} z\right)^{n / 2}}\right]
\end{gathered}
$$

for the partially localized case, and

$$
\begin{aligned}
V_{z \gg b} \rightarrow & E\left[1-\frac{z^{6-p}}{Q_{p}}\left(1-\frac{3 E Q_{F}}{2 m_{f} z^{6-p}}\right)\right] \\
& +\frac{J_{\perp}^{2} z^{10-2 p}}{2 m_{f} Q_{p}^{2}}\left(1-\frac{2 E Q_{F}}{m_{f} z^{6-p}}\right),
\end{aligned}
$$

for the delocalized case. Due to the presence of the $\mathrm{D} p$-brane, the usual repulsive potential of the source fundamental string (the part of the potential which is independent of $J_{\perp}$ ) gets an additional potential contribution from the source D $p$-brane. In the delocalized case [Eq. (52)], the repulsive force due to the probe fundamental string is completely "screened" by the source D $p$-brane and the probe feels the repulsive force due to the D $p$-brane, only. However, when the source fundamental string is localized at the source $\mathrm{D} p$-brane, the probe feels some repulsive contribution in the effective potential which signals existence of the source fundamental string. The (repulsive) centrifugal potential $\left(J_{\perp}\right.$ dependent term) on the probe is again suppressed due to the presence of the $\mathrm{D} p$-brane and is not repulsive anymore. [The term $J_{\perp}^{2} /\left(2 m_{f} H_{p}^{2} z^{2}\right)$ in Eq. (46) becomes a standard centrifugal potential term, if the source $\mathrm{D} p$-brane is absent, i.e., $H_{p}=1$.] In the case of the partially localized case, this term does not give rise to the force on the probe (ignoring the subleading $Q_{F}$ dependent term). However, when the source fundamental string is delocalized, the probe still feels a $J_{\perp}$ dependent attractive force (to the leading order, ignoring the subleading $Q_{F}$ dependent term).

At short distance $z \ll b$ from the source $\mathrm{D} p$-brane and very close to the source fundamental string $\left(x \ll \sqrt{Q_{p} b}\right)$, the effective potential is approximated to

$$
\begin{aligned}
V_{z \ll b, x \ll \sqrt{Q_{p} b}} \rightarrow & E-\frac{m_{f}}{2 Q_{p} Q_{F}} z\left(x^{2}+4 Q_{p} z\right)^{n / 2} \\
& +\frac{m_{f} J_{\perp}^{2}}{2 E^{2} Q_{p}^{2} Q_{F}^{2}}\left(x^{2}+4 Q_{p} z\right)^{n},
\end{aligned}
$$

for the partially localized case, and

$$
V_{z \ll b} \rightarrow E-\frac{m_{f}}{2 Q_{p} Q_{F}} z^{12-2 p}+\frac{m_{f} J_{\perp}^{2}}{2 E^{2} Q_{p}^{2} Q_{F}^{2}} z^{22-4 p},
$$

for the delocalized case. The usual ( $J_{\perp}$ independent) repulsive potential term due to the source fundamental string is suppressed by the contribution from the source $\mathrm{D} p$-brane. Again, the repulsive centrifugal potential is completely suppressed and becomes attractive.

We now study the motion of the probe along the radial direction $z$ of the overall transverse space. The turning point 
$z$, where the radial velocity $\dot{z}$ vanishes, of the probe's motion is given by the root of the following equation:

$$
1+\frac{\left(Q_{p} b\right)^{n / 2}}{2\left(x^{2}+4 Q_{p} z\right)^{n / 2}}=\frac{b_{*}^{2}}{Q_{p}} \frac{1}{z},
$$

where $b$ is given in Eq. (48) and $b_{*}=J_{\perp} / \sqrt{2 m_{f} E}$. In the delocalized case, the turning point $z$ satisfies the following equation:

$$
1+\frac{b^{6-p}}{2 z^{6-p}}=\frac{b_{*}^{2}}{Q_{p}} z^{4-p},
$$

where $b$ is given in Eq. (50). In the partially localized case, there is always one turning point at positive $z$ when $J_{\perp} \neq 0$, whereas there is no turning point for the $J_{\perp}=0$ case. In the delocalized case, when the angular momentum is nonzero, there is (i) one turning point at positive $z$ for $p<4$, (ii) one turning point at positive $z$ [no turning point] for a sufficiently large [small] value of $J_{\perp}$ for $p=4$, and (iii) one turning point at $z=b_{*}^{2} / Q_{p}-b / 2\left(b_{*}^{2}>Q_{p} b / 2\right)$ for $p=5$, whereas there is no turning point for the $J_{\perp}=0$ case. Furthermore, the force on the probe along the radial direction $z$, i.e., $F(z)$ $=-d V(z) / d z$, is always positive [vanishes] at $z=0$ when $x>0[x=0]$ in the partially localized case. In the delocalized case, the force always vanishes at $z=0$. So, the motion of the probe fundamental string along the $z$ direction can be summarized as follows. In the partially localized case, away from the source fundamental string $(x>0)$, the probe fundamental string will always bounce back as it approaches the source $\mathrm{D} p$-brane, but can be eventually absorbed by the source $\mathrm{D} p$-brane when the probe approaches the $\mathrm{D} p$-brane inside of the world volume of the source fundamental string $(x=0)$. In the delocalized case, the probe with $J_{\perp}=0$ will always be absorbed by the source $\mathrm{D} p$-brane. This seems to be due to the fact that the source fundamental string is delocalized on the source $\mathrm{D} p$-brane, i.e., is uniformly distributed over the world volume of the $\mathrm{D} p$-brane. When the probe has nonzero angular momentum $J_{\perp}$, the probe will bounce back as it approaches the $\mathrm{D} p$-brane for the following cases: (i) $p$ $<4$, (ii) the sufficiently large value of $J_{\perp}$ with $p=4$, and (iii) $b_{*}^{2}>Q_{p} b / 2$ with $p=5$. Otherwise, the probe will always be absorbed by the source as it approaches the $\mathrm{D} p$-brane.
[1] A.A. Tseytlin, Nucl. Phys. B487, 141 (1997).

[2] M. Douglas, J. Polchinski, and A. Strominger, J. High Energy Phys. 12, 003 (1997).

[3] I. Chepelev and A.A. Tseytlin, Phys. Rev. D 56, 3672 (1997).

[4] I. Chepelev and A.A. Tseytlin, Nucl. Phys. B515, 73 (1998).

[5] H. Liu and A.A. Tseytlin, J. High Energy Phys. 01, 010 (1998).

[6] A.A. Tseytlin and S. Yankielowicz, Nucl. Phys. B541, 145 (1999).

[7] N. Itzhaki, A.A. Tseytlin, and S. Yankielowicz, Phys. Lett. B 432, 298 (1998).

[8] D. Youm, Nucl. Phys. B556, 222 (1999).

[9] A. Fayyazuddin and D.J. Smith, J. High Energy Phys. 04, 030 (1999).

[10] A. Loewy, "Semilocalized brane intersections in SUGRA," hep-th/9903038.
[11] C.G. Callan and J.M. Maldacena, Nucl. Phys. B513, 198 (1998).

[12] G.W. Gibbons, Nucl. Phys. B514, 603 (1998).

[13] J. Gutowski and G. Papadopoulos, Phys. Lett. B 432, 97 (1998).

[14] D. Bak, J. Lee, and H. Min, Phys. Rev. D 59, 045011 (1999).

[15] J. Gutowski and G. Papadopoulos, Nucl. Phys. B551, 650 (1999).

[16] N.S. Manton, Phys. Lett. 110B, 54 (1982).

[17] M.F. Atiyah and N.J. Hitchin, Phys. Lett. 107A, 21 (1985).

[18] M.F. Atiyah and N.J. Hitchin, Philos. Trans. R. Soc. London A315, 459 (1985).

[19] G.W. Gibbons and P.J. Ruback, Phys. Rev. Lett. 57, 1492 (1986).

[20] R.C. Ferrell and D.M. Eardley, Phys. Rev. Lett. 59, 1617 (1987). 